\title{
ARTICLE OPEN \\ Chemically induced large-gap quantum anomalous Hall insulator states in III-Bi honeycombs
}

\author{
Christian P. Crisostomo ${ }^{1}$, Zhi-Quan Huang ${ }^{1}$, Chia-Hsiu Hsu ${ }^{1}$, Feng-Chuan Chuang $\mathbb{D}^{1,2}$, Hsin Lin ${ }^{3,4}$ and Arun Bansil ${ }^{5}$
}

The search for novel materials with new functionalities and applications potential is continuing to intensify. Quantum anomalous Hall (QAH) effect was recently realized in magnetic topological insulators (Tls) but only at extremely low temperatures. Here, based on our first-principles electronic structure calculations, we predict that chemically functionalized III-Bi honeycombs can support large-gap QAH insulating phases. Specifically, we show that functionalized AIBi and TIBi films harbor QAH insulator phases. GaBi and InBi are identified as semimetals with non-zero Chern number. Remarkably, TIBi exhibits a robust QAH phase with a band gap as large as $466 \mathrm{meV}$ in a buckled honeycomb structure functionalized on one side. Furthermore, the electronic spectrum of a functionalized TIBi nanoribbon with zigzag edge is shown to possess only one chiral edge band crossing the Fermi level within the band gap. Our results suggest that III-Bi honeycombs would provide a new platform for developing potential spintronics applications based on the QAH effect.

npj Computational Materials (2017)3:39; doi:10.1038/s41524-017-0044-9

\section{INTRODUCTION}

Two-dimensional (2D) topological materials have continued to gain increasing attention in the recent years. ${ }^{1-4}$ Among the large variety of possible topological phases, quantum anomalous Hall (QAH) insulators ${ }^{5}$ have drawn special interest since the QAH state, which supports chiral edge states, is highly suited for spintronics and low-power-consumption electronic applications. ${ }^{6-10}$ Unlike the quantum Hall state, which relies on the presence of an external magnetic field, the QAH state is realized through the effects of intrinsic spin-orbit coupling (SOC) and intrinsic magnetization in a material. ${ }^{11}$ The QAH state was first suggested by Haldane in 1988 using a tight-binding model on a honeycomb lattice, ${ }^{5}$ and it has been realized recently in magnetically doped $\mathrm{TI}$ thin films. ${ }^{11-15}$ However, all experimental realizations to date are limited to very low temperatures. It is important, therefore, to search for viable new materials that can support the QAH phase above room temperature, so that the applications potential of these materials can be developed.

Theoretical considerations suggest that the QAH effect should be generally achievable in TI thin films via magnetic order (e.g., ferromagnetism), which could be induced through magnetic doping or chemical functionalization. ${ }^{16}$ Given a quantum spin Hall (QSH) insulator, the QAH phase can be achieved by suppressing one of the two spin-channels via ferromagnetic (FM) ordering. ${ }^{7,17,18}$ Many studies have already shown that thin films of elements of groups IV, ${ }^{19,20} \mathrm{~V}_{1}^{21-23}$ and III-V ${ }^{24-29}$ harbor 2D QSH phases. In addition to the magnetic topological crystalline insulators, ${ }^{30,31}$ films of elements of groups IV ${ }^{16}$ and $V^{32-34}$ have also been predicted to harbor the QAH phases. Although it has been predicted that III-V films can support the QSH state in a number of freestanding ${ }^{24}$ and functionalized cases, ${ }^{25,26,28}$ only limited work has been reported toward the QAH phase ${ }^{35}$ with relatively small band gaps $(\sim 105 \mathrm{meV})$. It is highly desirable thus to realize a large gap 2D QAH phase in this group of materials as we attempt to do in this study.

Here we use first-principles calculations to predict new QAH insulator phases in III-Bi honeycombs where FM order ${ }^{13,18}$ is induced through chemical functionalization. In particular, since it has been shown previously that $\mathrm{N}$ atoms can support a net magnetic moment when adsorbed on a $\mathrm{TI}$ surface, ${ }^{36}$ we utilized two types of functionalization: (1) hydrogenation on both sides, and (2) decoration one side with $\mathrm{H}$ and with $\mathrm{N}$ on the other side. ${ }^{33}$ Our earlier work ${ }^{27}$ shows that the QSH phase in fully hydrogenated III-Bi honeycombs survives only up to two layers and that the system band gap is reduced as the III-V thin film gets thicker. For this reason, here we focus only on single-layer III-Bi films, which have a larger band gap compared to the multilayer films. We identify several QAH insulator phases with band gaps as large as $466 \mathrm{meV}$ by calculating the associated Chern numbers (Cs). We further confirm the presence of the QAH phase by calculating the edge states of functionalized TIBi nanoribbons. The proposed III-Bi films could be grown on suitable substrates and used in potential energy-efficient spintronics applications.

\section{RESULTS AND DISCUSSION}

Crystal structure of the 2D III-Bi honeycomb with adsorbed $\mathrm{N}$ and $\mathrm{H}$ atoms on the III-element and $\mathrm{Bi}$ sites [denoted as III-BiNH, Case I] and the related first Brillouin zone (BZ) with high-symmetry points are presented in Fig. 1, along with sideviews of the two-sided and one-sided functionalized planar (PL), buckled (BK), and inversely buckled (IBK) III-BiNH. We will also consider the alternate atomic

\footnotetext{
${ }^{1}$ Department of Physics, National Sun Yat-Sen University, Kaohsiung 804, Taiwan; ${ }^{2}$ Multidisciplinary and Data Science Research Center, National Sun Yat-Sen University, Kaohsiung 804, Taiwan; ${ }^{3}$ Centre for Advanced 2D Materials and Graphene Research Centre, National University of Singapore, Singapore 117546 , Singapore; ${ }^{4}$ Department of Physics, National University of Singapore, Singapore 117542, Singapore and ${ }^{5}$ Department of Physics, Northeastern University, Boston, MA 02115, USA

Correspondence: Feng-Chuan Chuang (fchuang@mail.nsysu.edu.tw) or Hsin Lin (nilnish@gmail.com)

Christian P. Crisostomo and Zhi-Quan Huang contributed equally to this work
}

Received: 13 March 2017 Revised: 28 August 2017 Accepted: 30 August 2017

Published online: 26 September 2017 


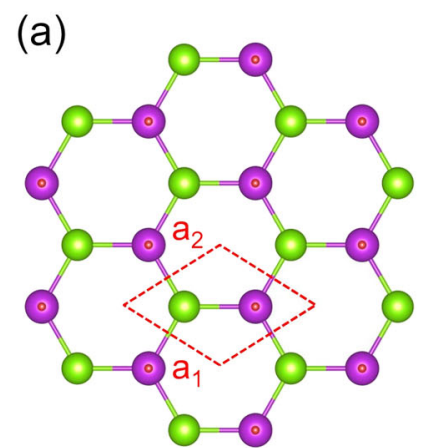

(C)

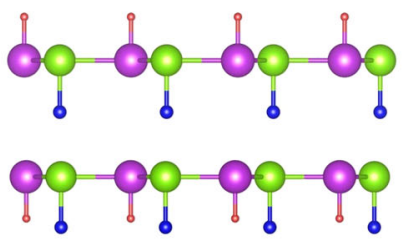

III

$\mathrm{Bi}$

$\begin{array}{ll}N & 0 \\ H & 0\end{array}$ (b)

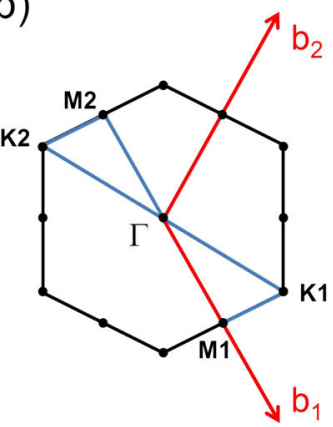

(e) (d)

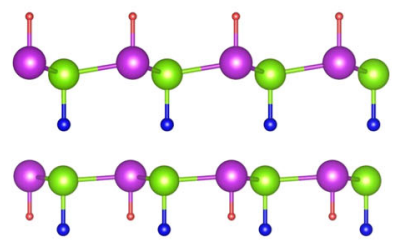

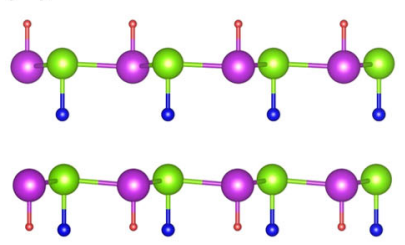

Fig. 1 a Crystal structure of III-Bi honeycomb with $\mathrm{N}$ and $\mathrm{H}$ adsorbed, respectively, on III-element and Bi atoms (III-BiNH). b The first Brillouin zone (BZ) with high-symmetry points. Sideviews of two-sidedly and one-sidedly functionalized III-BiNH in c planar (PL), d buckled (BK), and e inversely buckled (IBK) structure

\begin{tabular}{|c|c|c|c|c|c|}
\hline III-Bi & & $\begin{array}{l}\text { Lat. const. } \\
(\AA)\end{array}$ & $\begin{array}{l}\text { Total en. } \\
(\mathrm{eV})\end{array}$ & $\begin{array}{l}\text { Sys. gap } \\
\text { (meV) }\end{array}$ & Phase \\
\hline \multirow{4}{*}{$\begin{array}{l}\text { Case I (III- } \\
\text { BiNH) }\end{array}$} & $\mathrm{AlBi}$ & 4.946 & -14.34 & 48 & QAH \\
\hline & $\ln B i$ & 5.530 & -13.02 & -29 & $\mathrm{QAH}$ \\
\hline & $\mathrm{GaBi}$ & 5.117 & -13.46 & -43 & QAH \\
\hline & $\mathrm{TIBi}$ & 5.246 & -12.59 & 261 & $\mathrm{QAH}$ \\
\hline \multirow{4}{*}{$\begin{array}{l}\text { Case II (III- } \\
\text { BiHN) }\end{array}$} & $\mathrm{AlBi}$ & 4.881 & -14.35 & 52 & QAH \\
\hline & $\ln B i$ & 5.554 & -13.00 & -43 & $\mathrm{QAH}$ \\
\hline & $\mathrm{GaBi}$ & 5.119 & -13.46 & -43 & $\mathrm{QAH}$ \\
\hline & TIBi & 5.443 & -12.52 & 124 & QAH \\
\hline
\end{tabular}

configuration in which the $\mathrm{N}$ and $\mathrm{H}$ adsorption is on $\mathrm{Bi}$ and IIIelement instead, which is denoted as III-BiHN and will be referred to as Case II. Focusing on combinations of group III elements with $\mathrm{Bi}$ (III-Bi), which assume the QSH state over a range of lattice constants, we present in Table 1 the equilibrium lattice constant for the PL honeycomb, along with the associated total energies, system band gaps and topological invariants $(C \neq 0$ implies QAH state) for both cases I and II. Here the system band gap is defined as the energy difference between the conduction band minimum and the valence band maximum. Our calculations indicate that the system prefers (lower energy) the FM order over the nonmagnetic phase, and we found that both $\mathrm{AlBi}$ and TIBi possess a QAH insulator phase with band gaps of 48 and $261 \mathrm{meV}$, respectively, for Case I (52 and $124 \mathrm{meV}$ for Case II), while InBi and $\mathrm{GaBi}$ are both semimetals with $C=1$ in all cases.

Since TIBi exhibits a fairly large QAH band gap of $261 \mathrm{meV}$ and has also been shown to support a robust QSH phase in the freestanding as well as fully hydrogenated cases, ${ }^{24,26}$ we further explore its electronic structure in different $\mathrm{H}$ and $\mathrm{N}$ functionalizations. Figure 2 shows the electronic band structure of TIBiNH for adsorbed atoms on both sides [(a) PL and (d) IBK], and for the one-sided case [(b) PL and (e) BK] where we found a QAH phase with gap as large as $466 \mathrm{meV}$ for BK TIBiNH [Fig. 2e]. As for
TIBiHN [(c) and (f)], it harbors a QAH phase for the two-sided (PL and IBK) but not the one-sided functionalization. Both TIBiNH and TIBiHN are trivial insulators for two-sided functionalization in the BK structure. Important calculated parameters are summarized in Table 2 . Note that the inverse buckling distance is rather small $(0.144 \AA)$.

In order to gain further insight into the nature of the $\mathrm{QAH}$ phase, we investigated the two-side functionalized PL TIBiNH film as an exemplar system for exploring effects of SOC in both nonmagnetic and FM calculations more thoroughly. Figure 3 presents band structures for non-magnetic calculations without and with SOC, along with the corresponding FM results. We find this structure to support a net magnetic moment of $2 \mu_{\mathrm{B}}$ per unit cell, which is contributed mainly by $\mathrm{N}$ atoms. In the non-magnetic calculation, the system is metallic as seen in Fig. 3a, b. A net magnetic moment is induced through FM ordering upon $\mathrm{N}$ adsorption as seen in Fig. 3c (without SOC) with reference to the gapped spin-down (blue line) and gapless spin-up (red line) channels. Figure $3 d$ shows that when SOC is turned on in the FM calculation, it leads to the opening of an inverted gap involving $s$ and $p_{x, y}$ orbitals, and the QAH effect is realized.

Sufficient intrinsic magnetization is needed in order for a QSH-QAH transition to occur. Since $\mathrm{N}$ atoms carry most of the magnetic moment, it is interesting to explore evolution of the electronic structure with $\mathrm{N}$ coverage $\left(c_{\mathrm{N}}\right)$. For this purpose we will consider an augmented $2 \times 2$ supercell based on $\mathrm{PL}$ TIBiNH, which we found to have a lower total energy compared to TIBiHN. We start by fully passivating the TIBi film with $\mathrm{H}\left(\mathrm{C}_{\mathrm{H}}=1\right.$ or full hydrogen coverage) and then gradually replace, starting from one side, $\mathrm{H}$ by $N$ atoms until full $N$ coverage is achieved $\left(c_{N}=1\right)$. The lowest energy configuration is then chosen for further analysis. The resulting coverage-dependent band structures are shown in Fig. 4, along with information on system bandgaps and (total) magnetizations $(m)$ per unit cell as well as the topological phases assumed by the films. We found that fully hydrogenated TIBi (TIBiH2) [Fig. 4a] is a QSH insulator, which is consistent with our earlier study. ${ }^{26}$ Interestingly, already at $c_{N}=0.125$ [Fig. 4b], the system is seen to change from QSH to the QAH phase. This is to be contrasted with the results of a previous study ${ }^{33}$ of $\mathrm{PL} \mathrm{Bi}$ honeycombs with $\mathrm{H}$ and $\mathrm{N}$ adsorption where the critical point was reached only at $c_{N}=0.65$. We find the band gap to be 


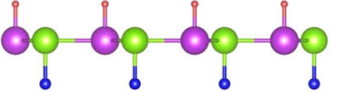

(a) $\mathrm{TIBiNH}(\mathrm{PL})$
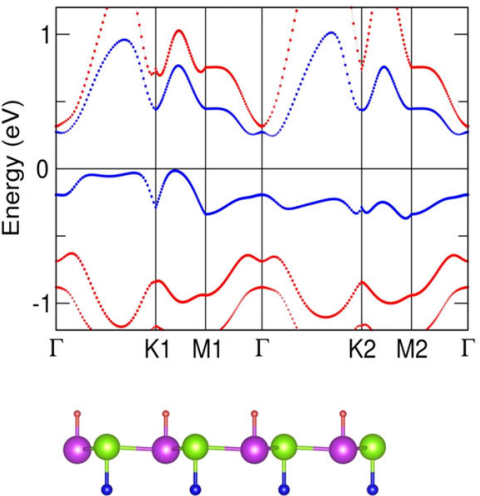

(d) $\mathrm{TIBiNH}$ (IBK)

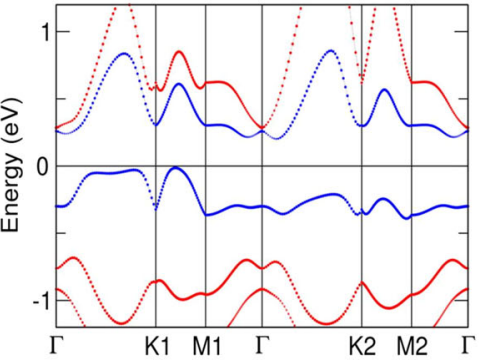

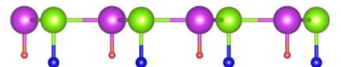

(b) TIBiNH (PL)
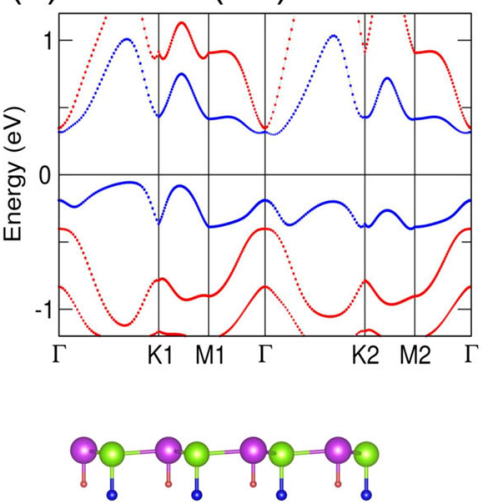

(e) TIBiNH (BK)

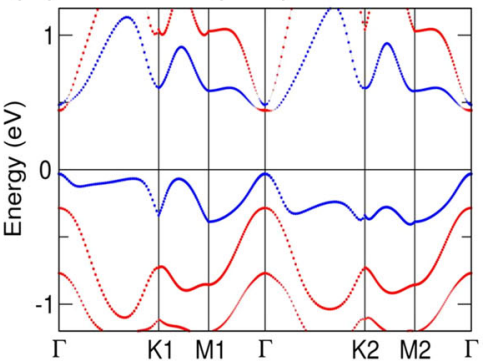

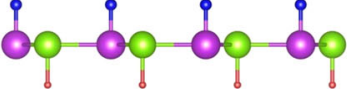

$\mathrm{TI}$

(c) TIBiHN (PL)

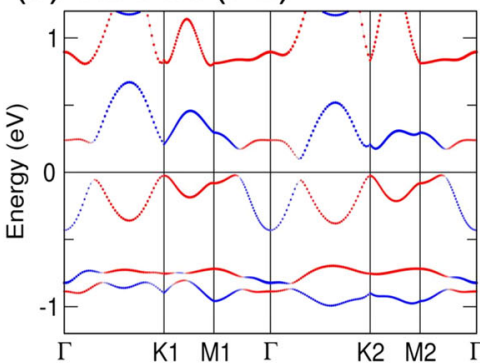

$\mathrm{Bi}$

$\mathrm{N}$

$\mathrm{H}$ o

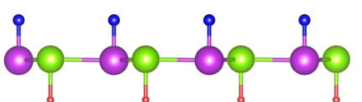

(f) $\mathrm{TIBiHN}$ (IBK)

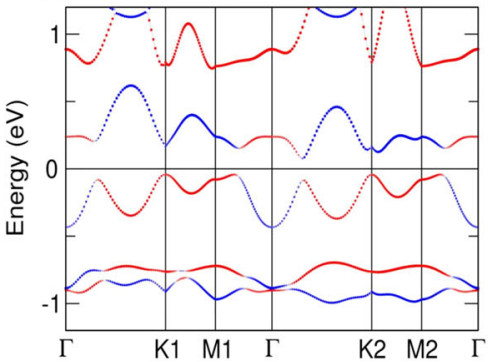

Fig. 2 Electronic band structure of TIBiNH film with two-sided adsorption for a planar (PL) and d inversely buckled (IBK) cases. The one-sided cases are considered in b for PL \& e for BK. c Planar and f IBK films. Red and blue circles show the $+s_{z}$ and $-s_{z}$ contributions, respectively

Tables 2. Calculated equilibrium lattice constants, total energies, system band gaps, and topological phases of TIBiNH (Case I) and TIBiHN (Case II) for two-sided and one-sided functionalizations

\begin{tabular}{|c|c|c|c|c|c|}
\hline & & Lat. const. ( $\AA$ ) & Total en. (eV) & Sys. gap (meV) & Phase \\
\hline \multicolumn{6}{|c|}{ TIBiNH (Case I) } \\
\hline \multirow[t]{2}{*}{ Both sides } & IBK & 5.258 & -12.52 & 220 & QAH \\
\hline & PL & 5.246 & -12.59 & 261 & $\mathrm{QAH}$ \\
\hline \multirow[t]{2}{*}{ One-side } & $\mathrm{BK}$ & 5.215 & -12.63 & 466 & QAH \\
\hline & PL & 5.224 & -12.55 & 350 & QAH \\
\hline \multicolumn{6}{|c|}{ TIBiHN (Case II) } \\
\hline \multirow[t]{2}{*}{ Both sides } & IBK & 5.452 & -12.50 & 161 & QAH \\
\hline & $\mathrm{PL}$ & 5.443 & -12.52 & 124 & QAH \\
\hline \multirow[t]{2}{*}{ One-side } & IBK & 4.867 & -12.89 & 295 & $\mathrm{NI}$ \\
\hline & PL & 5.404 & -12.62 & 69 & $\mathrm{NI}$ \\
\hline
\end{tabular}

remarkably large at 50 and $62.5 \% \mathrm{~N}$ coverage ( 258 and $245 \mathrm{meV}$ ). Combined with our earlier findings, ${ }^{26}$ where III-Bi honeycombs were predicted to exhibit robust, large-gap QSH phases, the present study further indicates a high degree of tunability of these films in the QAH regime. The full hydrogenation, fluorination, and their combination leads to the QSH phase of these films, while the adsorption of $\mathrm{H}$ and $\mathrm{N}$ (fluorine) on either side of the honeycomb induces crossover to the QAH (NI) phase.

Next, using the tight-binding Hamiltonians parameterized based on Wannier functions, we calculated the edge band spectrum of the PL TIBiNH film and compared it to that of the fully hydrogenated $\mathrm{TIBi}(\mathrm{TIBiH} 2)$ film. Ribbons with zigzag edges and sufficiently large width $(\sim 88 \AA)$ were constructed as shown in Fig. 5. The edge states (red and blue circles) of fully-hydrogenated and $\mathrm{H}$ and $\mathrm{N}$ functionalized TIBi films are presented in Fig. 5. Contributions of the left and right zigzag edges are proportional to the sizes of blue and red circles, respectively. In Fig. 5a, $\mathrm{TIBiH} 2$ exhibits a QSH state as shown by the odd number of band crossings of the Fermi level between $\pi / a$ and $\Gamma$ as well as between $-\pi / a$ and $\Gamma$, clearly indicating the presence of helical edge states. Moreover, we can see in Fig. $5 \mathrm{~b}$ that there is an odd number ( 3 for red bands and 1 for blue bands) of edge bands crossing the Fermi level between $-\pi / a$ and $\pi / a$ for each of the edges. The number of edge band crossings must be the same as the absolute value of the Chern number, which further confirms the presence of the $\mathrm{QAH}$ state in TIBiNH.

Chemical functionalization in III-Bi honeycombs could be achieved by growing these films on a suitable substrate or through the development of an appropriate experimental approach. Notably, some experimental studies have shown that ferromagnetism can be induced through chemical functionalization of graphene. ${ }^{37,38}$ Concerning experimental possibilities, in our previous studies, ${ }^{26,27}$ we have shown that fully hydrogenated III-Bi honeycombs could be viewed as a simplified sandwich structure. We further showed that by growing III-Bi on a substrate (e.g., GaBi on $\mathrm{Si}(111)$ ) and then passivating the other side with $\mathrm{H}$ does not result in a new phase compared to the case when the film is fully (functionalized) hydrogenated. In the case of the present III-Bi films, we may thus view $\mathrm{H}$ adsorption on one side of the honeycomb as a simple model of the substrate, while $\mathrm{N}$ atoms are used to saturate the dangling bond on the other side to induce 
(a) non-magnetic calculation

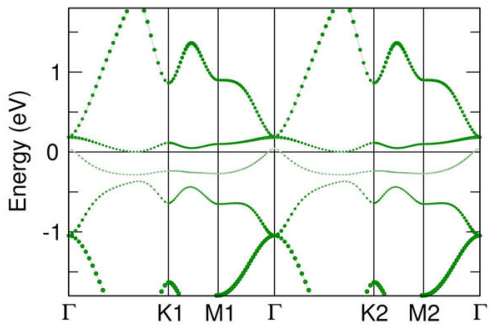

(c) ferromagnetic calculation

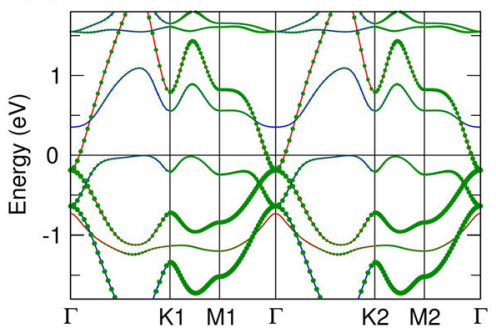

(b) non-magnetic calculation + SOC

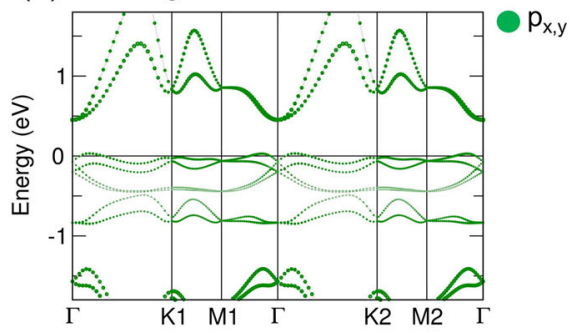

(d) ferromagnetic calculation + SOC

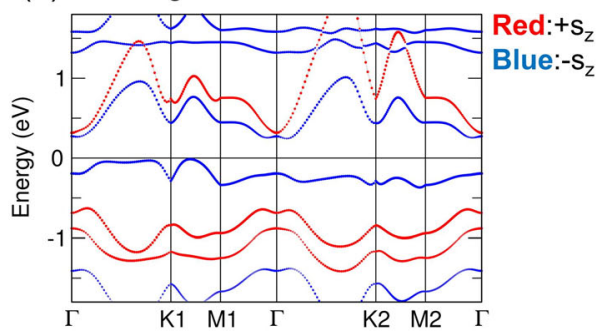

Fig. 3 Band structure of planar TIBiNH film in non-magnetic calculations a without and $\mathbf{b}$ with SOC. c, $\mathbf{d}$ are the band structures for ferromagnetic calculations without and with SOC, respectively. Green (red and blue) circles are proportional to the total $p_{x, y}\left( \pm s_{z}\right)$ contributions

(a) $c_{N}=0.0$

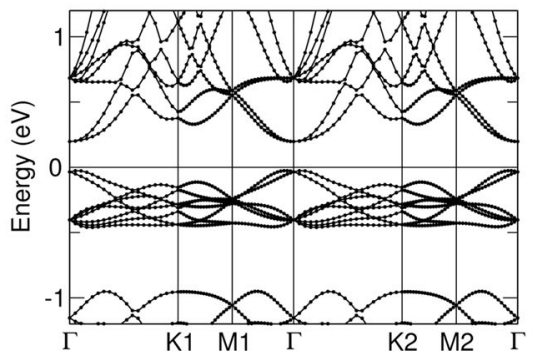

(d) $c_{N}=0.625$

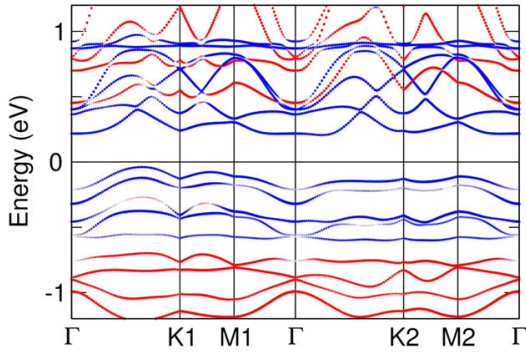

(b) $\mathrm{c}_{\mathrm{N}}=0.125$

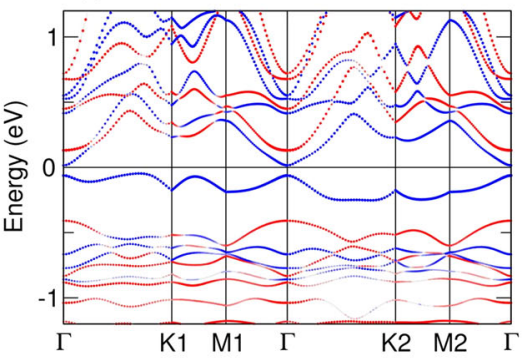

(e) $\mathrm{C}_{\mathrm{N}}=0.875$

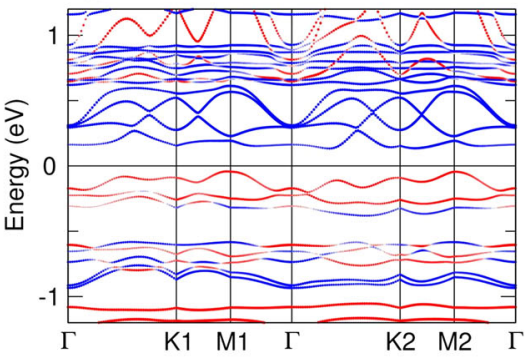

(c) $\mathrm{C}_{\mathrm{N}}=0.375$

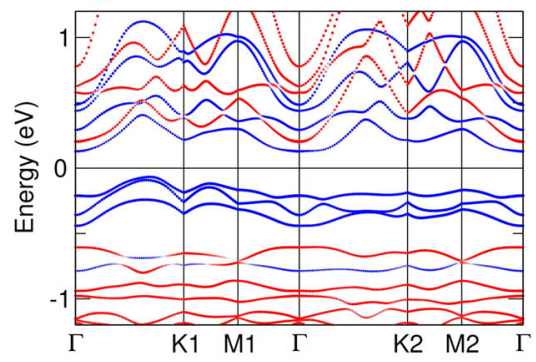

(f)

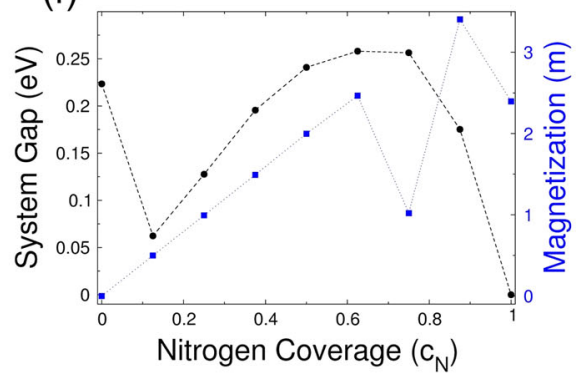

Fig. 4 Calculated band structures at $\mathrm{N}$ coverages of a 0.0 , b $0.125, \mathbf{c} 0.375$, d 0.625 , and e $0.875 \mathrm{ML}$. f System band gaps, total magnetizations (per unit cell), and phases of PL TIBiNH films for various coverages. Black (red and blue) circles in the band structures are proportional to the total s-orbital $\left( \pm s_{z}\right)$ contributions

the QAH phase, suggesting that our predictions are robust against substrate effects.

As to the choice of an appropriate substrate for our TIBi films, SiC (0001) or $\mathrm{Si}(111)$ would likely not be viable due to a substantial lattice mismatch. In this connection, we considered $\mathrm{CdTe}(111)$ as a substrate for supporting the TIBi film since it has a lattice constant of $4.687 \AA$. Two layers of $\mathrm{CdTe}(111)$ were used where the bottom Te atoms were passivated with $\mathrm{H}$, see Fig. 6 . We placed BiTl on the top surface. $\mathrm{Bi}$ is bonded to $\mathrm{Cd}$ atoms, and $\mathrm{Tl}$ atoms are then passivated with $\mathrm{N}$ atoms. All atoms were fully relaxed until the force on each atom was smaller than $0.001 \mathrm{eV} / \AA$. The distance between adjacent layers is found to be $2.89 \AA$. Since the optimized lattice constant of BK TIBiNH is $5.246 \AA$, N-TIBi film when placed on $\mathrm{CdTe}(111)$ would be compressively strained. FM band structures without and with SOC are presented in Fig. 6 and show that $\mathrm{N}-\mathrm{TIBi}$ on $\mathrm{CdTe}(111)$ is a semimetal with a system band gap of $-0.104 \mathrm{eV}$ and $C=2$, which is also the case for TIBiNH at this lattice constant.

Concerning the current experimental situation, a recent study ${ }^{39}$ demonstrated growth of $\mathrm{Tl}_{x} \mathrm{Bi}_{1-x}$ via $\mathrm{Bi}$ deposition on Tl-covered $\mathrm{Si}(111)$ substrate that resulted in different surface reconstructions. 
(a) QSH fully-hydrogenated TIBi

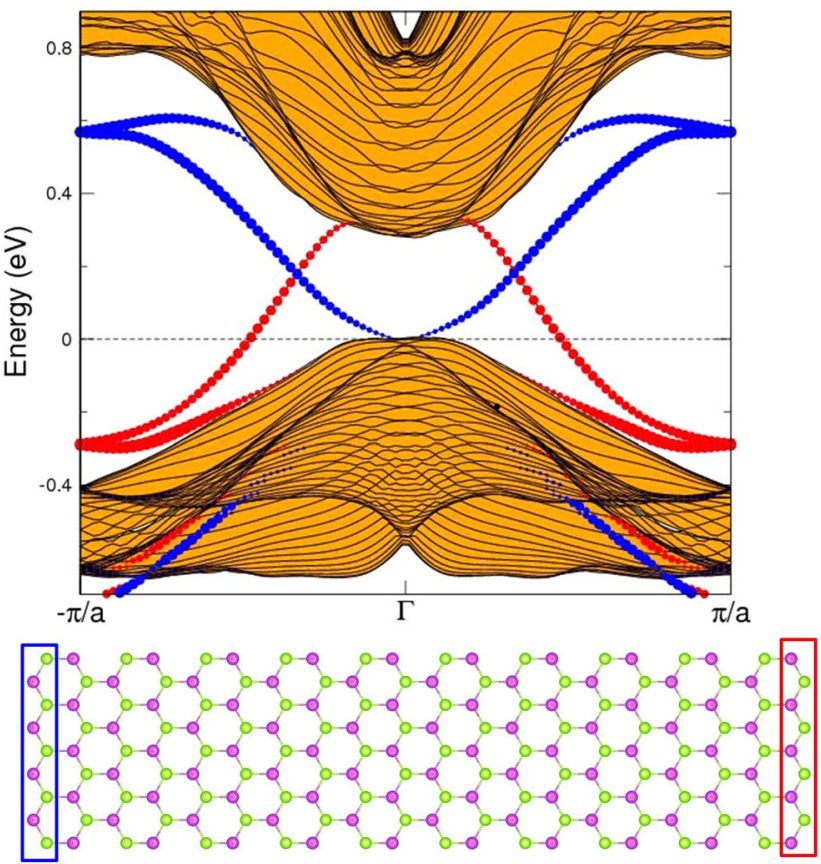

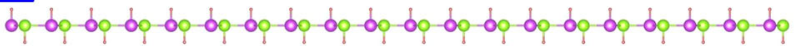

(b) QAH N and $\mathrm{H}$ functionalized TIBi

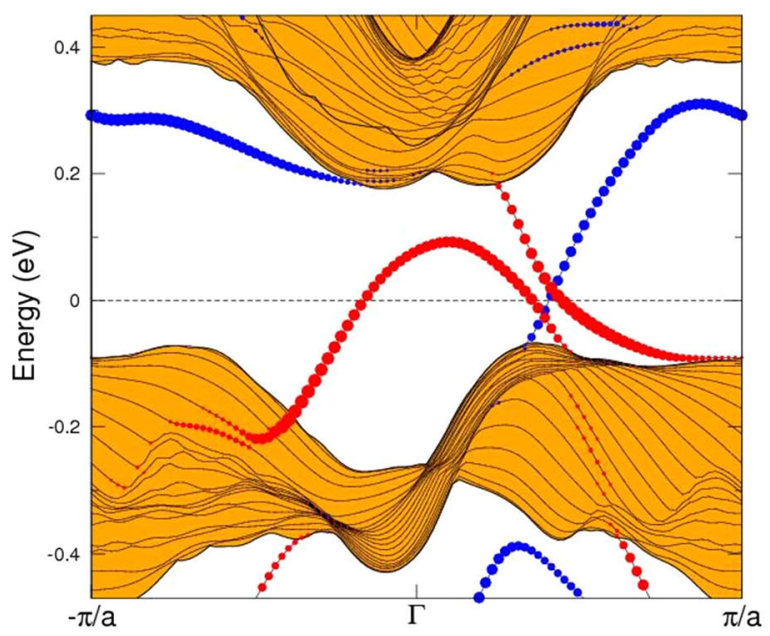

0
0
0
0

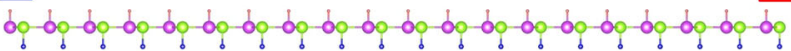

Fig. 5 Crystal and band structures along the zigzag edge of a fully hydrogenated and $\mathbf{b} \mathbf{H}$ and $\mathrm{N}$ functionalized planar TIBi film with $\boldsymbol{a}=5.246$ $\AA$. The orange-filled region denotes the bulk bands while contribution from the left (right) edge is marked with blue (red) circles

(a) Atomic structure of $\mathrm{N}-\mathrm{TIBi}$ on $\mathrm{CdTe}(111)$

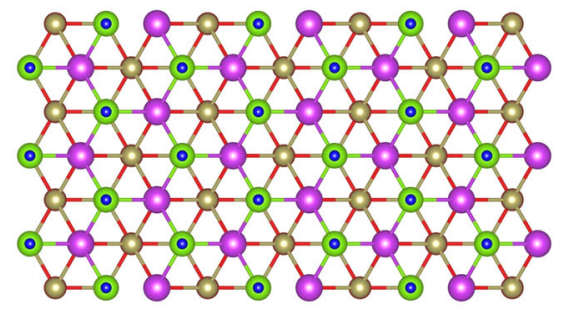

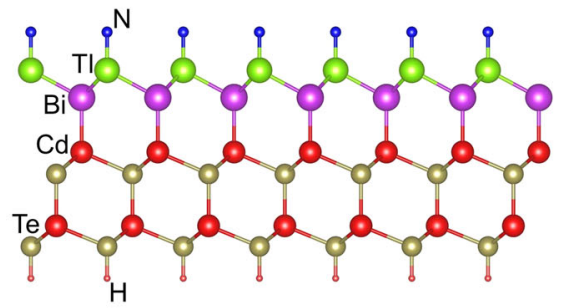

(b) Ferromagnetic calculation

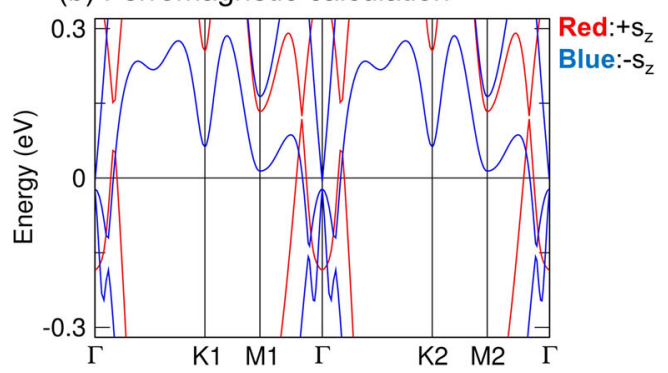

(c) Ferromagnetic calculation + SOC

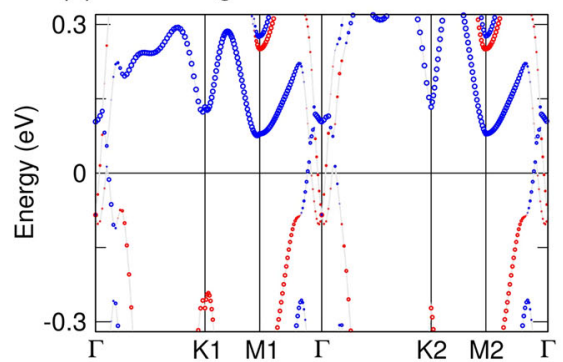

Fig. 6 a Top and side views of $\mathrm{N}$-functionalized TIBi on $\mathrm{CdTe}(111)$. The corresponding band structures $\mathbf{b}$ without and $\mathbf{c}$ with SOC are also shown. The red and blue lines/circles refer to spin-up and spin-down states, respectively

A related work realized honeycomb-like $\operatorname{InBi}$ on $\mathrm{Si}(111) .{ }^{40}$ Since our system is a perfect III-Bi honeycomb, this suggests that either a more advanced experimental technique needs to be developed or a more suitable substrate is needed for the perfect III-Bi honeycomb to be successfully synthesized.

We have systematically explored electronic and topological properties of functionalized III-Bi honeycombs for the purpose of identifying the existence of possible $\mathrm{QAH}$ insulator phases with large band gaps. TIBi films are found to exhibit a high degree of tunability for supporting QSH to the QAH phase via chemical adsorption of different functional groups. While symmetric and asymmetric functionalization using hydrogen and fluorine leads to the QSH phase, hydrogen and nitrogen adsorption induces the QAH phase. Our study suggests that functionalized TIBi films are a 
viable candidate material for exploiting the potential of the QAH effect towards spintronics applications.

\section{METHODS}

We performed our first-principles calculations within the density functional theory (DFT) framework utilizing the generalized gradient approximation. ${ }^{41-45}$ Projector-augmented-wave ${ }^{46}$ wave functions with energy cut-off of $400 \mathrm{eV}$ were used in the Vienna ab-initio simulation package. ${ }^{47,48}$ Crystal structures were optimized until the residual forces were no greater than $10^{-3} \mathrm{eV} / \mathrm{A}$. The self-consistency criteria for convergence was set at $10^{-6} \mathrm{eV}$. To simulate a thin film, a vacuum layer of at least $25 \AA$ along the $z$ direction was inserted. $\Gamma$-centered MonkhorstPack $^{49}$ grids of $24 \times 24 \times 1$ and $12 \times 12 \times 1$ were used for $1 \times 1$ and $2 \times 2$ honeycomb structures, respectively. We used maximally-localized Wannier functions provided by the WANNIER90 package. ${ }^{50}$ Wannier function based Hamiltonians were used to calculate Berry curvatures and edge states. In order to identify the topological phases, we calculated $\mathrm{Cs}^{51-53}$ by integrating the Berry curvature obtained using Kubo formula ${ }^{52,54}$ over the BZ. Moreover, we followed the method of ref. 55 for computing the $Z_{2}$ invariants. In this study, non-spin polarized calculations are referred to as non-magnetic, while FM spin-polarized calculations assume a FM configuration.

\section{Data availability}

The authors declare that all relevant data are available from the authors.

\section{ACKNOWLEDGEMENTS}

FCC acknowledges support from the National Center for Theoretical Sciences and the Ministry of Science and Technology of Taiwan under Grants Nos. MOST-104-2112-M110-002-MY3 and MOST-103-2112-M-110-008-MY3. He also thanks the support under NSYSU-NKMU JOINT RESEARCH PROJECT \#105-P005 and \#106-P005. He is also grateful to the National Center for High-performance Computing for computer time and facilities. The work at Northeastern University was supported by the US Department of Energy (DOE), Office of Science, Basic Energy Sciences grant number DE-FG02-07ER46352 (core research), and benefited from Northeastern University's Advanced Scientific Computation Center (ASCC), the NERSC supercomputing center through DOE grant number DE-AC02-05CH11231, and support (applications to layered materials) from the DOE EFRC: Center for the Computational Design of Functional Layered Materials (CCDM) under DE-SC0012575. H.L. acknowledge the Singapore National Research Foundation for support under NRF Award No. NRFNRFF2013-03.

\section{AUTHOR CONTRIBUTIONS}

F.C.C. and H.L. conceived and initiated the study. C.P.C, Z.Q.H., and C.H.H. performed first principles calculations. Z.Q.H. performed the Chern number calculation. C.P.C., C. H.H., Z.Q.H., F.C.C., H.L., and A. B. performed the detailed analysis and contributed to discussions. C.P.C., Z.Q.H, C.H.H, F.C.C., H.L., and A.B. wrote the manuscript. All authors reviewed the manuscript.

\section{ADDITIONAL INFORMATION}

Competing interests: The authors declare that they have no competing financial interests.

Publisher's note: Springer Nature remains neutral with regard to jurisdictional claims in published maps and institutional affiliations.

\section{REFERENCES}

1. Bansil, A., Lin, H. \& Das, T. Colloquium: topological band theory. Rev. Mod. Phys. 88, 021004 (2016).

2. Qi, X.-L. \& Zhang, S.-C. Topological insulators and superconductors. Rev. Mod. Phys. 83, 1057 (2011).

3. Hasan, M. Z. \& Kane, C. L. Colloquium: topological insulators. Rev. Mod. Phys. 82, 3045 (2010).

4. Nagaosa, N., Sinova, J., Onoda, S., MacDonald, A. H. \& Ong, N. P. Anomalous Hall effect. Rev. Mod. Phys. 82, 1539 (2010).

5. Haldane, F. D. M. Model for a quantum Hall effect without landau levels: condensed-matter realization of the "parity anomaly". Phys. Rev. Lett. 61, 2015 (1988).
6. Weng, H. et al. Quantum anomalous hall effect and related topological electronic states. Adv. Phys. 64, 227-282 (2015).

7. Qi, X.-L., Wu, Y.-S. \& Zhang, S.-C. Topological quantization of the spin Hall effect in two-dimensional paramagnetic semiconductors. Phys. Rev. B 74, 085308 (2006).

8. He, K., Wang, Y. \& Xue, Q.-K. Quantum anomalous Hall effect. Natl. Sci. Rev. 1, 38-48 (2014).

9. He, K. The quantum Hall effect gets more practical. Physics 8, 41 (2015).

10. Kou, X., Fan, Y., Lang, M., Upadhyaya, P. \& Wang, K. L. Magnetic topological insulators and quantum anomalous Hall effect. Solid State Commun 215, 34-53 (2015).

11. Chang, C.-Z. et al. High-precision realization of robust quantum anomalous Hall state in a hard ferromagnetic topological insulator. Nat. Mater. 14, 473-477 (2015).

12. Chang, C.-Z. et al. Thin films of magnetically doped topological insulator with carrier-independent long-range ferromagnetic order. Adv. Mater. 25, 1065-1070 (2013).

13. Chang, C.-Z. et al. Experimental observation of the quantum anomalous Hall effect in a magnetic topological insulator. Science 340, 167-170 (2013).

14. Bestwick, A. J. et al. Precise quantization of the anomalous Hall effect near zero magnetic field. Phys. Rev. Lett. 114, 187201 (2015).

15. Kou, X. et al. Metal-to-insulator switching in quantum anomalous Hall states. Nat. Commun. 6, 8474 (2015).

16. Wu, S.-H., Shan, G. \& Yan, B. Prediction of near-room-temperature quantum anomalous Hall effect on honeycomb materials. Phys. Rev. Lett. 113, 256401 (2014).

17. Liu, C.-X., Qi, X.-L., Dai, X., Fang, Z. \& Zhang, S.-C. Quantum anomalous Hall effect in $\mathrm{Hg}_{1-y} \mathrm{Mn}_{y} \mathrm{Te}$ quantum wells. Phys. Rev. Lett. 101, 146802 (2008).

18. $\mathrm{Yu}, \mathrm{R}$. et al. Quantized anomalous Hall effect in magnetic topological insulators. Science 329, 61-64 (2010).

19. $\mathrm{Xu}$, Y. et al. Large-gap quantum spin hall insulators in tin films. Phys. Rev. Lett. 111, 136804 (2013).

20. Chou, B.-H. et al. Hydrogenated ultra-thin tin films predicted as two-dimensional topological insulators. New. J. Phys. 16, 115008 (2014).

21. Liu, C. C. et al. Low-energy effective Hamiltonian for giant-gap quantum spin Hall insulators in honeycomb X-hydride/halide ( $\mathrm{X}=\mathrm{N}-\mathrm{Bi})$ monolayers. Phys. Rev. B 90, 085431 (2014).

22. Song, Z. et al. Quantum spin hall insulators of $B i X / S b X(X=H, F, C l$, and $B r)$ monolayers with a record bulk band gap. NPG Asia Mater. 6, e147 (2014).

23. Chen, L. et al. Edge state modulation of bilayer Bi nanoribbons by atom adsorption. Phys. Chem. Chem. Phys. 16, 17206 (2014).

24. Chuang, F.-C. et al. Prediction of large-gap two-dimensional topological insulators consisting of bilayers of group III elements with Bi. Nano Lett. 14, 2505-2508 (2014).

25. Ma, Y. et al. Two-dimensional inversion-asymmetric topological insulators in functionalized III-Bi bilayers. Phys. Rev. B 91, 235306 (2015).

26. Crisostomo, C. P. et al. Robust large-gap two-dimensional topological insulators in hydrogenated III-V bilayers. Nano Lett. 15, 6568-6574 (2015).

27. Yao, L.-Z. et al. Predicted growth of two-dimensional topological insulator thin films of III-V compounds on Si(111) substrate. Sci. Rep. 5, 15463 (2015).

28. Li, L., Zhang, X., Chen, X. \& Zhao, M. Giant topological nontrivial band gaps in chloridized gallium bismuthide. Nano Lett. 15, 1296-1301 (2015).

29. Zhao, M., Chen, X., Li, L. \& Zhang, X. Driving a GaAs film to a large-gap topological insulator by tensile strain. Sci. Rep. 5, 8441 (2015).

30. Fang, C., Gilbert, M. J. \& Bernevig, B. A. Large-chern-number quantum anomalous Hall effect in thin-film topological crystalline insulators. Phys. Rev. Lett. 112, 046801 (2014).

31. Zhang, F., Li, X., Feng, J., Kane, C. L. \& Mele, E. J. Zeeman field-tuned transitions for surface chern insulators. Preprint at https://arxiv.org/abs/1309.7682 (2013).

32. Liu, C.-C., Zhou, J.-J. \& Yao, Y. Valley-polarized quantum anomalous Hall phases and tunable topological phase transitions in half-hydrogenated Bi honeycomb monolayers. Phys. Rev. B 91, 165430 (2015).

33. Jin, K.-J. \& Jhi, S.-H. Quantum anomalous Hall and quantum spin-Hall phases in flattened Bi and Sb bilayers. Sci. Rep. 5, 8426 (2015).

34. Zhou, T., Zhang, J., Zhao, B., Zhang, H. \& Yang, Z. Quantum spin-quantum anomalous Hall insulators and topological transitions in functionalized $\mathrm{Sb}(111)$ monolayers. Nano. Lett. 15, 5149-5155 (2015).

35. Chen, S.-P. et al. Prediction of quantum anomalous Hall Insulator in halffluorinated GaBi honeycomb. Sci. Rep. 6, 31317 (2016).

36. Jin, K.-H. \& Jhi, S.-H. Effect of atomic impurities on the helical surface states of the topological insulator Bi2Te3. J. Phys. Condens. Matter 24, 175001 (2012).

37. Hong, J. et al. Room-temperature magnetic ordering in functionalized graphene. Sci. Rep. 2, 624 (2012).

38. Giesbers, A. J. M. et al. Interface-induced room-temperature ferromagnetism in hydrogenated epitaxial graphene. Phys. Rev. Lett. 111, 166101 (2013). 
39. Gruznev, D. V. et al. Synthesis of two-dimensional $\mathrm{Tl}_{x} \mathrm{Bi}_{1-x}$ compounds and Archimedean encoding of their atomic structure. Sci. Rep. 6, 19446 (2016).

40. Denisov, N. V. et al. Bismuth-indium two-dimensional compounds on Si(111) surface. Surf. Sci. 651, 105-111 (2016)

41. Hohenberg, P. \& Kohn, W. Inhomogeneous electron gas. Phys. Rev. 136, B864 (1964).

42. Kohn, W. \& Sham, L. J. Self-consistent equations including exchange and correlation effects. Phys. Rev. 140, A1133 (1965).

43. Ceperley, D. M. \& Alder, B. J. Ground state of the electron gas by a stochastic method. Phys. Rev. Lett. 45, 566 (1980).

44. Perdew, J. P. \& Zunger, A. Self-interaction correction to density-functional approximations for many-electron systems. Phys. Rev. B 23, 5048 (1981).

45. Perdew, J. P., Burke, K. \& Ernzerhof, M. Generalized gradient approximation made simple. Phys. Rev. Lett. 77, 3865 (1996).

46. Kresse, G. \& Joubert, D. From ultrasoft pseudopotentials to the projector augmented-wave method. Phys. Rev. B 59, 1758 (1999).

47. Kresse, G. \& Hafner, J. Ab initio molecular dynamics for liquid metals. Phys. Rev. $B$ 47, 558 (1993)

48. Kresse, G. \& Furthmüller, J. Efficient iterative schemes for an initio total-energy calculations using plane-wave basis set. Phys. Rev. B 54, 11169 (1996).

49. Monkhorst, H. J. \& Pack, J. D. Special points for Brillouin-zone integrations. Phys. Rev. B 13, 5188 (1976).

50. Mostofi, A. A. et al. wannier90: a tool for obtaining maximally-localised Wannier functions. Comput. Phys. Commun. 178, 685-699 (2008).

51. Qiao, Z. et al. Quantum anomalous Hall effect in graphene from Rashba and exchange effects. Phys. Rev. B 82, 161414 (2010).
52. Thouless, D. J., Kohmoto, M., Nightingale, M. P. \& den Nijs, M. Quantized Hall conductance in a two-dimensional periodic potential. Phys. Rev. Lett. 49, 405 (1982)

53. Kohmoto, M. Topological invariant and the quantization of the Hall conductance. Ann. Phys. N.Y. 160, 343 (1985).

54. Yao, Y. et al. First principles calculation of anomalous Hall conductivity in ferromagnetic bcc Fe. Phys. Rev. Lett. 92, 037204 (2004).

55. Fukui, T. \& Hatsugai, Y. J. Quantum spin Hall effect in three dimensional materials: Lattice computation of $Z_{2}$ topological invariants and its application to $\mathrm{Bi}$ and $\mathrm{Sb}$. Phys. Soc. Jpn. 76, 053702 (2007).

Open Access This article is licensed under a Creative Commons cc) Attribution 4.0 International License, which permits use, sharing, adaptation, distribution and reproduction in any medium or format, as long as you give appropriate credit to the original author(s) and the source, provide a link to the Creative Commons license, and indicate if changes were made. The images or other third party material in this article are included in the article's Creative Commons license, unless indicated otherwise in a credit line to the material. If material is not included in the article's Creative Commons license and your intended use is not permitted by statutory regulation or exceeds the permitted use, you will need to obtain permission directly from the copyright holder. To view a copy of this license, visit http://creativecommons. org/licenses/by/4.0/.

(c) The Author(s) 2017 